

RESEARCH COMMUNICATION

\title{
The fluorescent detection of Paradiplozoon sp. (Monogenea: Diplozoidae) attachment clamps' sclerites and integumental proteins
}

\author{
S.J. MILNE and A. AVENANT-OLDEWAGE* \\ Department of Zoology, Kingsway Campus, University of Johannesburg \\ P.O. Box 524, Auckland Park, 2006 South Africa
}

\begin{abstract}
MILNE, S.J. \& AVENANT-OLDEWAGE, A. 2006. The fluorescent detection of Paradiplozoon sp. (Monogenea: Diplozoidae) attachment clamps' sclerites and integumental proteins. Onderstepoort Journal of Veterinary Research, 73:149-152

In the genus Paradiplozoon, few hard structures are present therefore most of the taxonomic studies have focused on the unique ultrastructure of the sclerites. Alcohol-fixed specimens were transferred to BSA-saline for $5 \mathrm{~min}$. before staining indefinitely with $5 \mu \ell$ WGA-TRX, $5 \mu \ell$ SYTO 9 and $5 \mu \ell$ of Calcofluor White M2R. Rhodamine, Fluorescein and DAPI bandpass filters on the microscope enabled selective light wavelength illumination of the three flourochromes by a mercury light source. This method provided an easy and rapid methodology to show the internal sclerites of attachment clamps. It is suitable for alcohol preserved specimens and may have additional applications in other helminth organisms.
\end{abstract}

Keywords: Fluorescence, integument, Monogenea, Paradiplozoon, sclerites

Paradiplozoon (Monogenea: Diplozoidae) is an ectoparasitic helminth of yellowfish, Labeobarbus aeneus within the Vaal Dam that is situated in the Gauteng province of South Africa (26ㄷ' S; $28^{\circ} 10^{\prime}$ E). The monogenean gill parasite occurs as two hermaphroditic individuals fused in permanent cross copula. Le Brun, Renaud \& Lambert (1988) pointed out that there is a lack of valid morpho-anatomic criteria for determining species of the closely related genus Diplozoon as they lack sclerified genitalia which are less subjective to adaptive pressures than the haptor. They also found that four species of Diplozoon in Southern France could be separated by differing allozyme electrophoretic mobilities.

\footnotetext{
* Author to whom correspondence is to be directed Accepted for publication 15 March 2006-Editor
}

Most of the taxonomic studies on the Diplozoidae have focused on the unique ultrastructure of the sclerites situated in the attachment clamps that are positioned in two rows of four each on opposing sides of the organism's opisthaptor. The usage of standard techniques, such as trichrome stains and scanning electron microscopy (SEM) have limitations when observing the sclerites. In scanning electron micrographs the bivalve-like structure of the attachment clamps is clearly visible. However, due to the apical lamina of the tegument covering the clamps, there is limited visibility of the chitinous sclerites. Therefore, fluorochromy was employed to improve the visibility of the parasite's micromorphology.

Chitin is an acetate of the polysaccharide that contains glycosamine as its most important constituent. Chitin and various chitinases are widely distributed throughout the animal kingdom as structural com- 
Fluorescent detection of Paradiplozoon sp. attachment clamps
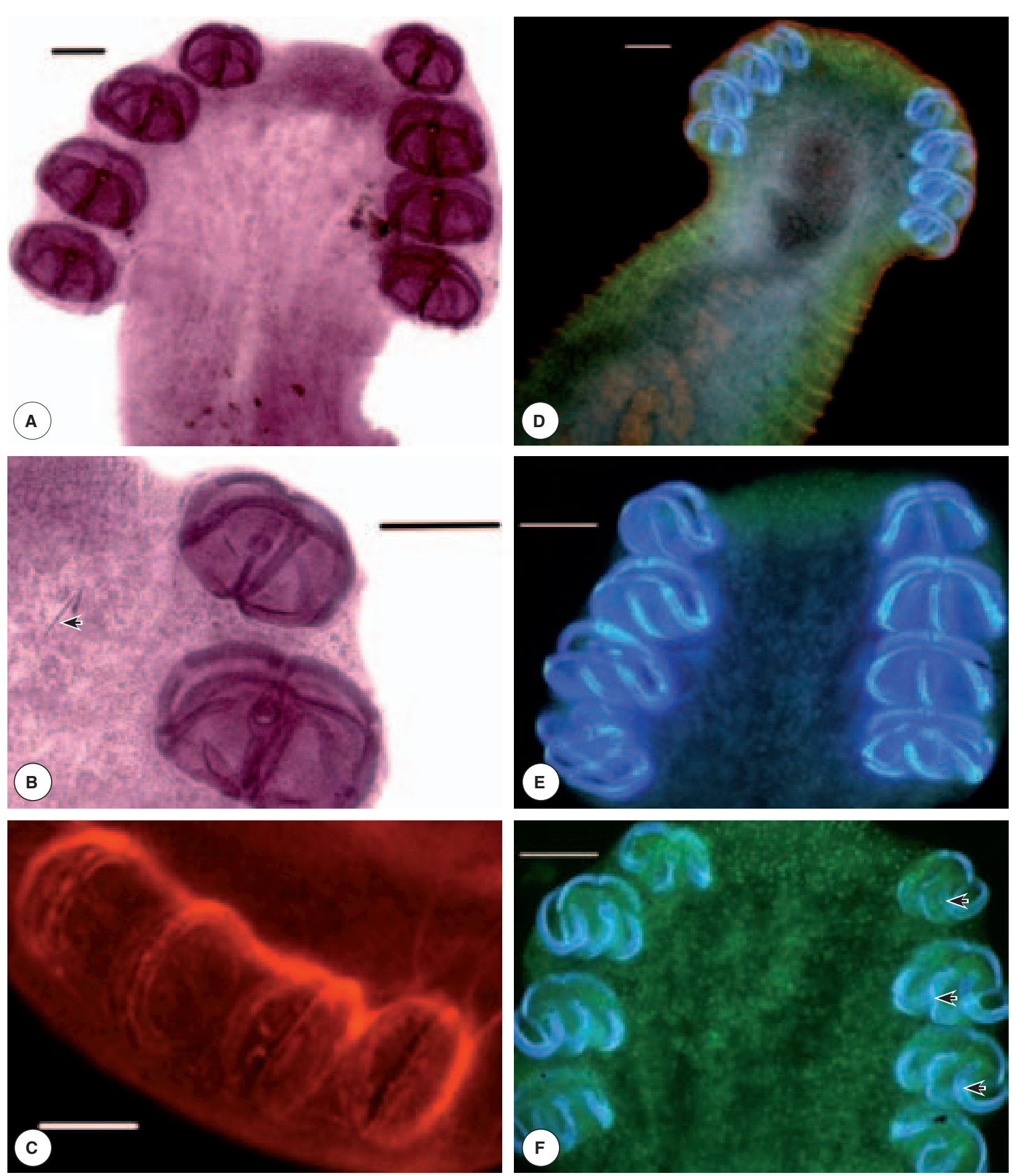

FIG. 1 Photomicrographs of Paradiplozoon morphology. Scale bars $=100 \mu \mathrm{m}$

A Dorsal view of opisthaptor, showing clamp morphology. Hörens trichrome stain

B Dorsal view of opisthaptor, arrow indicates position of hamilus. Hörens trichrome stain

C Photomicrograph of fluorescing glycocalyx after staining with WGA-1 Texas red

D Photomicrograph following staining with composite fluorochromes

E Dorsal view showing detail morphology of sclerites following staining with fluorochromes

F Ventral view showing detail morphology of sclerites following staining with fluorochromes, arrows indicate position of thickening in the anterior jaw 


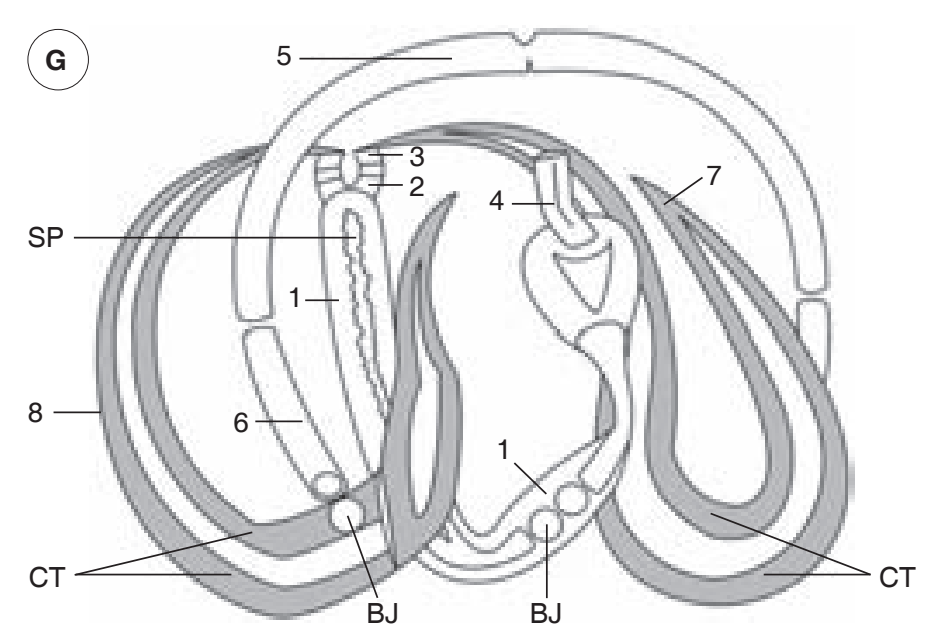

FIG. 1 Photomicrographs of Paradiplozoon morphology (cont.)

$G$ Diagram of the second clamp on the left, showing the anatomy of the clamp sclerites following fluorochrome staining

1 Median plate

2 Trapeze spur

3 Joining sclerites of the median plate

4 Sclerites of the distal tip of the median plate

5 Median sclerite of the posterior jaw

6 Lateral sclerite of the posterior jaw

7 Spiky spur

8 anterior jaw

BJ Ball joint

CT Chitinous thickening

SP Serrated median plate

ponents of invertebrates' exoskeletons, eukaryotic cells and bacteria. Chitin consists of $\mathrm{N}$-acetyl-Dglucosamine residues that are joined by $B(1 \rightarrow 4)$ glucosidic linkages (Voet, Voet \& Pratt 1999). It was therefore decided to conduct the morphological study of Paradiplozoon clamps using indirect fluorochromy for the visualization of the organism's integument. Direct fluorochromy was also conducted on the organism as a nuclear counter stain to ascertain the general morphology of the organism.

Yellowfishes were collected from the Vaal Dam using $90 \mathrm{~mm}$ mesh size gill nets. The fish were killed by severing the spinal cord. The gills were subsequently removed, placed in dam water and scrutinized utilizing a dissection microscope. The 27 parasites collected were individually removed from the gill filaments and transferred to a drop of dam water on a glass slide. A cover slip, separated from the slide by petroleum jelly, was then placed onto the first to ensure that each parasite was fixed in a flat position. The two cohesive slides impounding the parasite were transferred to Petridishes containing $70 \%$ ethanol for $10 \mathrm{~min}$, thereby killing the organism. Thereafter each parasite was preserved in $70 \%$ ethanol.

The "Cell Culture Determination Kit" protocol (Molecular Probes, Eugene, Oregon.) was followed as the fluorochrome kit was designed to test for gram positive bacteria and the technique was applied for helminths for the first time. Working solutions were prepared according to the instructions and stored at $-20^{\circ} \mathrm{C}$. The preserved Paradiplozoon were transferred onto glass slides and covered by $10 \mu \ell$ of BSAsaline for $5 \mathrm{~min}$ before staining with the addition of $5 \mu \ell$ WGA-TRX, $5 \mu \ell$ SYTO 9 and $5 \mu \ell$ of Calcofluor White M2R indefinitely. A cover slip was placed over each specimen and sealed with nail varnish. The specimens were subsequently viewed and photographed with a Zeiss Axioplan 2 epi-illumination fluorescence microscope, operated by Axiovision 4.3 software. Rhodamine, Fluorescein and DAPI bandpass filters on the microscope enabled selective light wavelength illumination of the three flourochromes by a mercury light source.

Specimens stained with Horen's trichrome (Fig. 1A) show that the general morphology of the parasite is highlighted but that the visibility of the sclerites in the clamps is limited. It is evident that each clamp consists of a pair of hinged jaws supported by median and lateral sclerites (Ramsamy, Brennan \& Halton 1995). At a high magnification the hamuli, which are residual larval hooks, can be observed (Fig. 1B).

The Paradiplozoon specimens were observed to autofluoresce when excited with UV light (365 nm) produced by an Hg lamp source and DAPI bandpass filter. The chemical composition of monogenean attachment clamps is ambiguous and it is unknown whether they are made up of keratin, chitin, quinine-tanned protein or collagen (Roberts \& Janovy 2005). However, a strong blue autofluorescence was observed under UV irradiation in hookworm hooklets, which are known to be made of chitin (Rost 1995) indicating that the sclerites in Paradiplozoon probably also contain chitin; which is in contrast to the finding of Lyons (1966), who stated that the sclerites of monogeneans are composed of scleroproteins and not the glycoprotein; chitin. Comparing the sclerites of the clamps as shown in Fig. 1E, 1F and $1 G$ it is observed that much of the detail of the clamp morphology occurs on the posterior side of the clamp. In Fig. 1E and 1F the chitinised components show up in three dimensions, highlighting the 
hinges and joints in the sclerites which are not visible in trichrome staining (Fig. 1A and 1 B). The chitinised thickening, the ball joint of the lateral sclerite attached to the anterior jaw and the three chitinised joints in the anterioir part of the median plate becomes visible with this technique (Fig. 1F and 1G).

The direct localization of the nucleic acids (antigen) of the Paradiplozoon was done utilizing the fluorescent SYTO 9 green nucleic acid stain (antibody). The SYTO 9 fluorochrome penetrates the compromised plasma membranes of eukaryotic cells labelling the DNA and RNA with a 1000-fold fluorescence enhancement upon nucleic acid binding (Haugland 2002). This is observed in Fig. 1D-F. It provides a counterstain to the WGA-Texas Red-X, Calcofluor White M2R providing a point of reference for the general morphology of the organism. WGATexas Red is specific for $N$-acetyl-B-D-glucosamine, a constituent glucosaminyl residue of chitin as well as $\mathrm{N}$-acetylneuraminic acid (sialic acid) and $\mathrm{N}$-acetyl muraminic acid which are component carbohydrates of the organisms glycocalyx (Johnsen, Hasner, Schnell \& Wuertz 2000). To support this further it was found that two mannose-rich glycoproteins similar to those previously mentioned, are found in the glycocalyx of Gyrodactylus derjavini (Monogenea) (Buchmann \& Lindenstrøm 2002). These mannoserich glycoproteins are important antigens and stimulants of the host's alternative complement pathway (Whittington, Cribb, Hamwood \& Halliday 2000).

The glycocalyx of the anucleate tegument fluoresced red as shown in Fig. 1C. WGA-Texas Red is a lectin bound fluorochrome that binds to the sialic acid compounds present on the apical lamina of the paradiplozoon's tegument, demonstrating the specific binding of antibodies to antigens. This proves that the glycocalyx of this Paradiplozoon is made up of $N$-acetylneuraminic acid and $\mathrm{N}$-acetyl muraminic acid, contrasting Lyons' (1966) finding that it consists of scleroproteins.

This technique provides an easy and rapid methodology to show the internal sclerites and the anatomy of attachment clamps. It is suitable for alcohol preserved specimens and may have additional applications for other helminth organisms.

\section{ACKNOWLEDGEMENTS}

We thank the NRF and the University of Johannesburg for funding and infrastructure. Dr Richard Greenfield and Ms Gina Walsh are thanked for technical assistance during field work.

\section{REFERENCES}

BUCHMANN, K. \& LINDENSTRØM, T. 2002. Interaction between monogenean parasites and their fish hosts. International Journal for Parasitology, 32:309-319.

HAUGLAND, R.P. 2002. Handbook of fluorescent probes and research products, $9^{\text {th }}$ edition. Oregon: Molecular Probes.

JOHNSEN, A.R. HASNER, M. SCHNELL, A. \& WUERTZ, S. 2000. Evaluation of fluorescently lectins for noninvasive localization of extracellular polymeric substances in Sphigomonas biofilms. Applied and Environmental Microbiology, 66: 3487-3491.

LE BRUN, N., RENAUD, F. \& LAMBERT, A. 1988. The genus Diplozoon (Monogenea, Polyopisthocotylea) in southern France: speciation and specificity. International Journal for Parasitology, 18:395-400.

LYONS, M. 1966. The chemical nature and evolutionary significance of monogenean attachment sclerites. Parasitology, 56: 63-100.

RAMASAMY, P., BRENNAN, G.P. \& HALTON, D.W. 1995. Ultrastructure of the surface structures of Allodiscocotyla diacanthi (Polyopistoctylea: Monogenea) from the gills of the marine teleost fish, Scomberoides tol. International Journal for Parasitology, 25:43-54.

ROBERTS, L.S. \& JANOVY, J. 2005. Foundations of Parasitology. New York: Mc Graw-Hill.

ROST, F.W.D. 1995. Fluorescence microscopy. Vol 2, Cambrige: Cambridge University Press.

VOET, D., VOET, J.G. \& PRATT, C.W. 1999. Fundamentals of biochemistry. Hoboken: John Wiley \& Sons, Inc.

WHITTINGTON, I.A., CRIBB, B.W., HAMWOOD, T.E. \& HALLIDAY, J.A. 2000. Host-specificity of monogean (platyhelminth) parasites: a role for anterior adhesive areas? International Journal for Parasitology, 3:305-320. 